\title{
Acinic Cell Carcinoma of the Salivary Gland with Metastatic Spread to the Pancreas
}

\author{
Jessica L. Geiger ${ }^{a}$ Joaquín J. García ${ }^{b}$ Katharine A.R. Price \\ Departments of ${ }^{\mathrm{a} I n t e r n a l ~ M e d i c i n e ~ a n d ~}{ }^{\mathrm{b}}$ Laboratory Medicine and Pathology, and \\ 'Division of Medical Oncology, Mayo Clinic Rochester, Rochester, Minn., USA
}

\section{Key Words}

Acinic cell carcinoma · Pancreas · Metastasis · Salivary cancer

\begin{abstract}
Metastatic disease to the pancreas is rare among solid tumors and has not been well described for salivary cancers. We report a patient who developed an isolated metastatic lesion in the pancreas from acinic cell carcinoma of the salivary gland, presenting as acute pancreatitis.

(c) 2014 S. Karger AG, Basel
\end{abstract}

\section{Introduction}

Salivary gland carcinomas are relatively uncommon, accounting for approximately $6 \%$ of all malignancies of the head and neck and $<1 \%$ of all newly diagnosed cancers in the United States annually [1]. The World Health Organization describes 24 distinct malignant epithelial tumors of salivary origin [2]. Mucoepidermoid carcinoma, adenoid cystic carcinoma, and adenocarcinoma account for over $75 \%$ of all salivary gland carcinomas, as described by the Memorial Sloan-Kettering experience [3]. Acinic cell carcinoma (AcCC) is a less common subtype comprising $6-10 \%$ of all salivary gland tumors $[3,4]$. AcCCs are typically well differentiated and rarely develop distant metastases. We report a case of locally recurrent AcCC that developed an isolated metastasis to the pancreas.

Katharine A.R. Price, MD

Division of Medical Oncology, Mayo Clinic Rochester

200 First Street SW

Rochester, MN 55905 (USA)

E-Mail price.katharine@mayo.edu 
Geiger et al.: Acinic Cell Carcinoma of the Salivary Gland with Metastatic Spread to the Pancreas

\section{Case Presentation}

In 2005, a 46-year-old woman presented with several years of progressive left jaw pain and swelling and ultimately developed partial left-sided facial paralysis. In August 2005, she underwent a left total parotidectomy with partial sacrifice of the left facial nerve. Histopathologic examination of the deep left parotid gland showed a thinly encapsulated lowgrade salivary gland neoplasm. Tumor cells exhibited round to oval nuclei with inconspicuous nucleoli and basophilic granular cytoplasm that is characteristic of well-differentiated AcCC. Surgical margins were negative. In April 2008, the patient developed a local recurrence in the left neck, confirmed by fine needle aspiration. On May 29, 2008, she underwent surgical excision of the left neck mass with negative margins and one sampled lymph node negative for disease. No other treatment modalities were given. Two years later, the patient developed a second local recurrence, and in June 2010, a $5 \times 3$-mm mass consistent with recurrent AcCC was removed from the posterior triangle. In October 2010, a surveillance PET/CT scan revealed a 1-cm hypermetabolic nodule between the left styloid process and the tip of the mastoid. Fine-needle aspiration findings were consistent with a third local recurrence of AcCC. In January 2011, the patient had a left posterior neck exploration surgery with left neck dissection. Pathology revealed a single focus of recurrent disease in a lymph node measuring $2.6 \mathrm{~cm}$. Surgical margins were negative but narrow at $<0.1 \mathrm{~cm}$. Three other lymph nodes sampled were negative for metastatic disease. The patient did not receive any adjuvant therapy and was placed on expectant observation.

In August 2011, the patient presented to our institution for a second opinion regarding management of a fourth local recurrence presenting as an enlarging, asymptomatic neck mass below the left mandible. On physical exam, a single $1-\mathrm{cm}$ non-tender nodule was palpable just below the mandible, overlying the left sternocleidomastoid muscle. She had no other palpable masses or lymphadenopathy present. An MRI scan of the face and neck revealed a $1.3-\mathrm{cm}$ mass in the previous operative bed in addition to a 1.4- $\mathrm{cm}$ enhancing mass at the skull base. CT of the chest, abdomen, and pelvis showed no evidence of distant metastatic disease. On August 29, 2011, the patient had a wide local excision of a locally recurrent AcCC measuring $1.5 \times 1.3 \times 1.1 \mathrm{~cm}$ (fig. 1a). Margins were negative. The second skull base mass seen on imaging was not amenable to surgical resection. The patient subsequently received concurrent chemoradiation therapy to the left neck from October through December 2011 with a total dose of 7,000 cGy in 35 fractions with weekly cisplatin. A surveillance PET/CT scan performed in March 2012 showed no evidence of disease.

In August 2012, the patient was admitted to a local hospital for epigastric pain, nausea, and vomiting, and was diagnosed with acute pancreatitis. An outside MRI scan of the abdomen revealed a mass in the head of the pancreas (fig. 2). Esophagogastroduodenoscopy and an endoscopic ultrasound with biopsy of the mass were performed, and pathology revealed a well-differentiated neoplasm consistent with metastatic AcCC of salivary gland origin. A PET/CT scan showed minimal fluorodeoxyglucose avidity corresponding with the pancreatic mass but no other areas of active disease and no evidence of local recurrence in the head and neck. On September 21, 2012, the patient underwent a laparoscopic metastasectomy. Microscopic examination revealed a $3.0-\mathrm{cm}$ lobulated neoplasm set within a bed of desmoplastic stroma of the pancreatic neck (fig. 1b). These tumor cells were morphologically compatible with the excised primary and recurrent tumors resected. Moreover, an immunohistochemical stain for trypsin (Meridian Life Science, Inc., Memphis, Tenn., USA) was negative, essentially ruling out the possibility of acinar cell carcinoma of the pancreas and confirming the diagnosis of metastatic AcCC to the pancreas. Perineural invasion was present. Surgical margins were negative. Multiple (24) regional lymph nodes were negative 
for metastatic disease. The patient recovered well and resumed routine surveillance. To date, she has had no evidence of disease progression.

\section{Discussion}

To our knowledge, this is the first report of a pancreas metastasis from AcCC of the salivary gland. Despite multimodality treatment, salivary gland malignancies can recur locoregionally or distantly. For slower-growing histologies such as AcCC, recurrences can occur after a delayed length of time, not uncommonly past the standard five-year follow-up period [5]. Therefore, lifelong surveillance and follow-up is recommended. Distant metastatic disease may occur in salivary gland malignancies, with the best data coming from studies of adenoid cystic carcinoma. The most common site of distant metastasis is the lung [6], followed by bone and liver $[7,8]$. Other reported metastatic sites include the cerebrum, thyroid, and spleen [7, 8]. A report from 1979 describes a case of adenoid cystic carcinoma metastasizing to the skin [9]. Overall, metastases to the pancreas are uncommon in solid tumors, with autopsy studies showing pancreatic involvement in widely metastatic disease in $3-12 \%$ of all patients with diffuse metastatic disease [10]. The most common solid tumors to metastasize to the pancreas include carcinomas of the kidney, lung, breast, colon, stomach, esophagus, and melanoma [11]. Metastatic spread to the pancreas has not been well described for salivary cancers. As chemotherapy plays a limited role in the treatment of salivary cancers, even in the palliative setting, surgery is often a mainstay of treatment. Given the isolated metastatic site and the prolonged natural history of AcCC, surgical resection was a good therapeutic option for our patient.

\section{Conclusion}

Distant metastatic disease can occur years after the initial diagnosis of AcCC. Although rare, metastatic spread to the pancreas can occur and should be kept in the differential diagnosis as clinically indicated.

\section{Disclosure Statement}

The authors declare that there are no conflicts of interest.

\section{References}

1 American Cancer Society: Cancer facts and figures. 2013.

http://www.cancer.org/research/cancerfactsstatistics/index.

2 World Health Organization Classification of Tumours. International Agency for Research on Cancer (IARC): Pathology and Genetics of Head and Neck Tumours. Lyon, IARC Press, 2005.

3 Spiro RH: Salivary neoplasms: overview of a 35-year experience with 2,807 patients. Head Neck Surg 1986;8:177-184.

4 Hoffman HT, Karnell LH, Robinson RA, Pinkston JA, Menck HR: National Cancer Data Base report on cancer of the head and neck: acinic cell carcinoma. Head Neck 1999;21:297-309.

5 Chandana SR, Conley BA: Salivary gland cancers: current treatments, molecular characteristics and new therapies. Expert Rev Anticancer Ther 2008;8:645-652.

6 Hamper K, Lazar F, Dietel M, et al: Prognostic factors for adenoid cystic carcinoma of the head and neck: a retrospective evaluation of 96 cases. J Oral Pathol Med 1990;19:101-107. 


\section{Case Reports in Oncology}

\begin{tabular}{l|l}
\hline Case Rep Oncol 2014;7:195-198 \\
\hline DOI: 10.1159/000360395 & $\begin{array}{l}\text { ○ 2014 S. Karger AG, Basel } \\
\text { www.karger.com/cro }\end{array}$ \\
\hline
\end{tabular}

Geiger et al:: Acinic Cell Carcinoma of the Salivary Gland with Metastatic Spread to the Pancreas

7 Bradley PJ: Distant metastases from salivary glands cancer. ORL J Otorhinolaryngol Relat Spec 2001;63:233-242.

8 van der Wal JE, Becking AG, Snow GB, van der Waal I: Distant metastases of adenoid cystic carcinoma of the salivary glands and the value of diagnostic examinations during follow-up. Head Neck 2002;24:779-783.

-9 Vinod SU, Gay RM: Adenoid cystic carcinoma of the minor salivary glands metastatic to the hand. South Med J 1979;72:1483-1485.

10 Rumancik WM, Megibow AJ, Bosniak MA, Hilton S: Metastatic disease to the pancreas: evaluation by computed tomography. J Comput Assist Tomogr 1984;8:829-834.

11 Adsay NV, Andea A, Basturk O, Kilinc N, Nassar H, Cheng JD: Secondary tumors of the pancreas: an analysis of a surgical and autopsy database and review of the literature. Virchows Arch 2004;444:527-535.
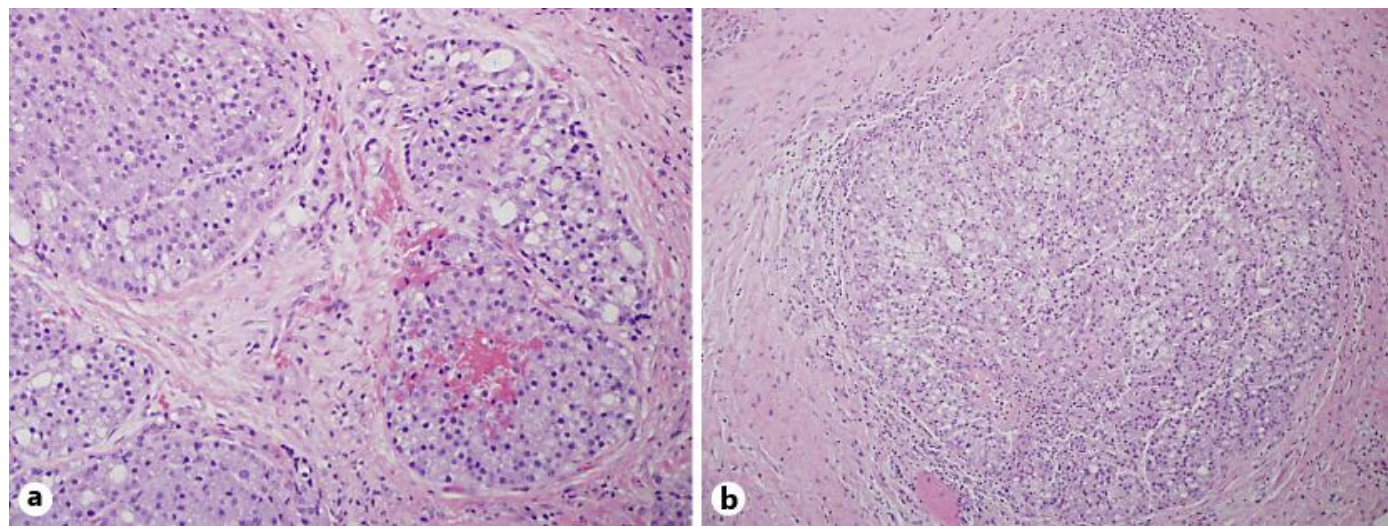

Fig. 1. a Intermediate-power examination of the recurrent salivary gland tumor in the left neck (H\&E. $\times 100$ ). The tumor cells are relatively monotonous, show round to oval nuclei, and basophilic granular cytoplasm, characteristic of well-differentiated AcCC. b Intermediate-power examination of the metastatic salivary gland tumor in the head of the pancreas (H\&E. $\times 100)$. Similar to the primary salivary gland tumor, the tumor cells are relatively monotonous and exhibit basophilic granular cytoplasm that is characteristic of well-differentiated AcCC.

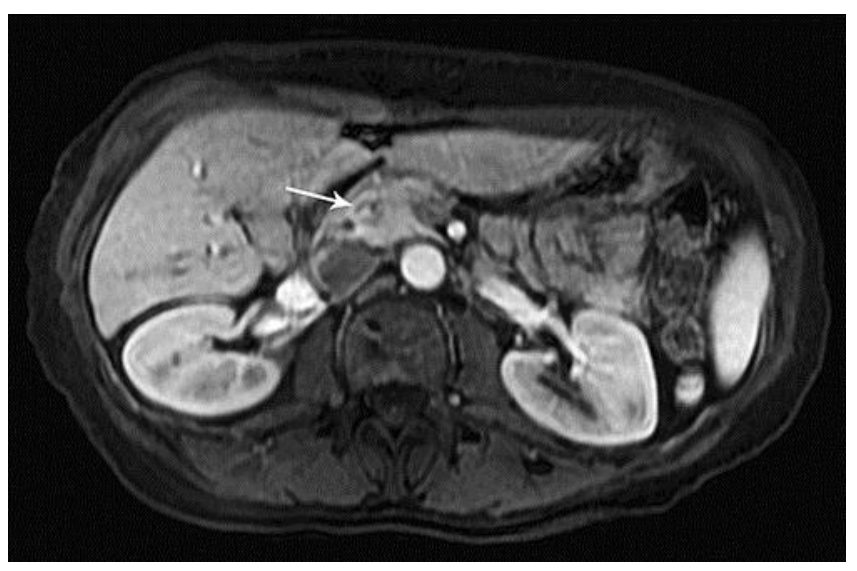

Fig. 2. An MRI scan with and without gadolinium with the arrow indicating a 9-mm hypoenhancing lesion in the pancreas. 